\title{
Style Features of Late Romantic Musical Texture
}

\author{
Oksana Sheludyakova \\ Ural Conservatoire \\ Yekaterinburg, Russia \\ E-mail: k046421@yandex.ru
}

\begin{abstract}
In the article by O.I. Scheludjakova the texture as the leading aspect of development of late melos is investigated. Peculiarities on different levels of relationship among melody and accompanying voices, rhythmic and modal organization, pattern, and harmony are reduced. The texture is analyzed as method of exposition, as deployment mode, as the element of dynamic infusion, factor of "emotions specifications". The subject of attention in the article is melodical utterances of late romanticism epoch in their complexity in multidimensionality. Various manifestations of texture in the utterance were studied, a systematics of the most characteristic types of texture for the late Romantic era was proposed, special attention was paid to the melodization of texture.
\end{abstract}

Keywords-musical texture; melodic; late Romantic style; textured melody functions

\section{INTRODUCTION}

The texture of Late Romanticism has a vivid originality. Even its external parameters amaze us:

- the number of voices, texture ideas, and sometimes layers sharply increases;

- the texture complexity increases, a variety of mixed harmonies appear;

- register range increases, tessitura expands;

- an intensification of the melos occurs, the number of independent melodies increases - two or more simultaneities are possible;

- melodization of accompanied voices occurs.

The musical language of the era may be considered an encyclopedia of texture techniques: all harmonies, all types of presentation with varying degrees of completeness are used we find amazing ingeniousness in the interpretation of musical tissue. The methods of many past eras are revived typically Late Romantic findings are emerging on their basis.

Moreover, each textural type is perceived not only as a musical and linguistic method with a certain imaginative potential, but also as some kind of a symbol. Thus, a Baroque fugal imitative polyphony can appear in the works of Late Romanticism (for example, in Liszt's Sonata h-moll, Tchaikovsky's Symphony No. 4 and 6, etc.) as a dramatic process of a theme development, a reflection of the complexity of intellectual processes, the multiline development of the idea given. The style of Viennese Classicism is often associated with the clarity, balance and harmony of the inner world: it will be enough to recall the main theme of the first part of Mahler's Symphony No. 4, some parts of Tchaikovsky's Symphonic Suites and Taneyev's Quartets in which the picture of the ideal of higher harmony and order are resurrected.

Texture can become the object of theatricalization. In this sense, the melody as a theatrical hero is emphasized with the help of a spatial location (highest register, remoteness from other voices), through "spotlight flashing" (emphasizing by dynamic means, for example, $\boldsymbol{f}$ against the background of $\boldsymbol{m} \boldsymbol{p}$ other voices), or silence of other "characters". The streaks and characteristics of the "hero" are often hyperbolized, underlined by special convexity, and sometimes by the sharpness of the instruments: for example, when applied to a melody, it can be bright bounces, dissonances, modeling of speech through agogics, traits, etc.

In other cases, the texture serves the psychologization of the character, the internal justification of a given technique, the creation of the effect of emotion, state, and process of thinking development. It becomes possible primarily due to the perception of the melody itself as the author's voice, confession, and, accordingly, consideration of the rest of the texture through the prism of the "character" (or author) and his environment. "It is not the colorfulness of the sound, not the association with the external "acoustic" space, but the depth of the inner psychological "space" reflected through the functional and logical movement and development of the textural components are in this case, perceived as the main thing [1].

Sometimes the textual ideas were associated with the philosophical conceptions of the universe. Samples of monody and heterophony sounded as a reflection of the idea of conciliarity, unity, and sometimes severe archaism. The chord harmony was perceived as one of the expressions of the idea of unity in diversity, integrity and organization; subvoice polyphony - as a simultaneous statement and outpouring of close, kindred spirits. Finally, the homophonic harmony was the best evidence of clarity in the "distribution of roles": the hero's statements and less significant and sometimes secondary background were isolated. 


\section{Processes of Melodization of Musical TeXture}

The most important texture ${ }^{1}$ tendency in the considered period is the melodization of the entire musical material. Depending on the specific textural conditions, this tendency finds expression in the appearance of various new harmonies based on the original textural model.

- Melodization leads to an increase in the number of melodic lines.

- Melodization of accompanied, background voices causes the emergence of a mixed, homophonic and polyphonic harmony.

- Melodic transformation of a chord harmony helps its polyphony:

a) formation of the sub-voice and polyphonic type texture;

b) reinterpretation of externally unchanged chord texture: pairwise voices coordination (a kind of analogue of a monorhythmic counterpoint of a strict style), combining the development processes along the vertical, horizontal and diagonal lines (by identifying counterpoint shifted motives in single voices).

Moderate sounds (pedals) in Late Romanticism are often colored with a melodic ornament or rhythmic figuration. In many works the polyphonic textural cells are constantly repeated which gives the sound a special fullness and delicacy.

Similar changes occur with duplications which grow into supporting voices and autonomous voices from pure heterophony. The general tendency towards melodization of the entire musical material is reflected in the increase in the number of accompanied counterpoint lines.

Here's an example. The polymelodic texture of the theme from Mahler's Symphony No. 9 is formed from isolated motives and syntagmas dispersed around the musical material and various instrumental groups, in "Fig. 1":
In the texture analysis the concept of "texture cell" is used (E. Nazaykinskiy). It means "a relatively complete area of textural development during which all the elements specific for a given type of texture are fully appearing, usually followed by repetition of a textural configuration pattern" $[294$, p. 80$]$.

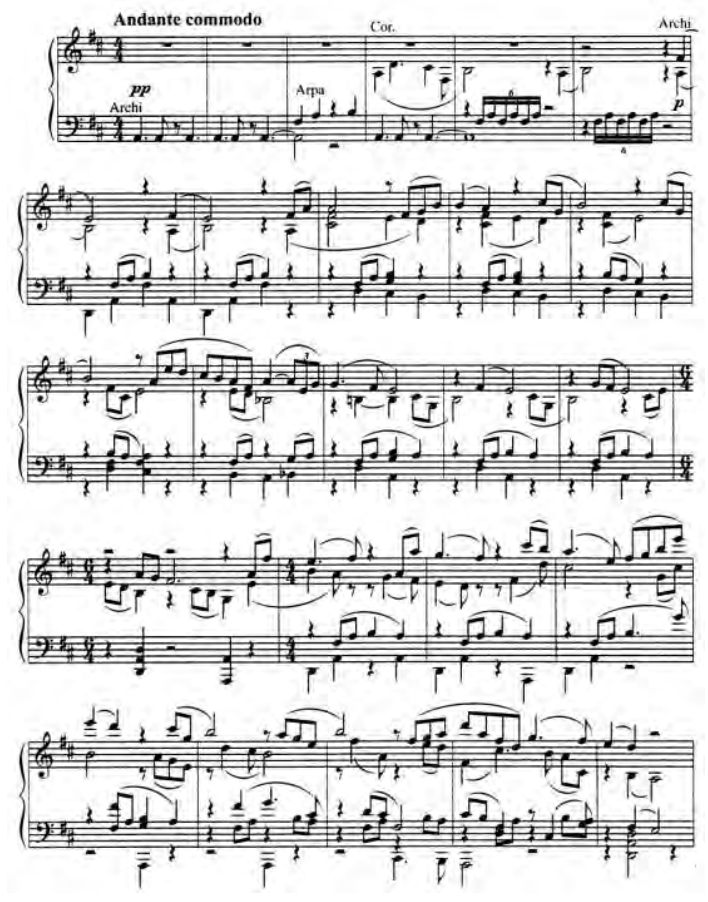

Fig. 1. G. Mahler. Symphony No. 9. P. I.

All of them can be considered as variants of a single intonational idea, and the theme as a whole can be interpreted as a textural and melodic variations in the authentic turn in which the smooth unsteadiness-steadiness rocking forms a picture of serene tranquility, a wise harmony of being. "Theme with the essence of the infinity of renewals, the infinity of turns and illuminations unfolds in the continual change of variations" [2]. Here the texture layering is combined with the ultimate "dispersion" of the melodic line into "atoms"-motives. Brief motives-impulses are quite simple and connected by complementarity principle: trichord intonations, tertiary moves, perfect fourth based motives, seconds allow personification of intervals and lead to a significant fractionality of the statement.

The degree of melodization of the entire material is amazing: each voice is interpreted as melodic, intensive processes of polyphonic "coloring" of homophonic material due to the imitative development of the simplest figures. It is important that in this statement both the principle of melodic polyphony (imposition of several melodic lines) and the complementary and counterpoint method (brief thematic idea shift along the diagonal) are combined. In this case, there is no perception of "viscosity" because the simplicity of intonational ideas, the logicality and regularity in their evolution give a melodic richness to the sound without making it heavy.

The completeness of such statements is formed in the relationship of melodic lines, and their totality. Paradoxically, under the conditions of a homophonic harmony, the laws of fugal forms operate - tension increase coincides with the accumulation of a number of melodic voices and, on the contrary, the dramatic emptying process is accompanied by a decrease in their number of sounding in simultaneity. We 
should emphasize that it is not about a simple increase of the texture density, but about imposition of autonomous melodic lines.

\section{THE MAIN VARIETIES OF TEXTURE}

The emergence of several melodies naturally leads to the emergence of a polyphonic harmony. The most important role of polyphony in the era of Late Romanticism is unquestionably not accidental. Firstly, the texture polyphony indirectly serves as a reflection of the consciousness polyphony. The complex processes of internal dialogue and the polylogue could not be reflected within the traditional homophonic harmony. Secondly, the Late Romanticism mission of a unique summing up of the musical art development inevitably led to the richest treasury of polyphonic technique which played such a significant role in the history of music. Thirdly, the search for bizarre "heterogeneous mixtures" (Novalis) to reflect the world of poetic dreams also could not do without combining the signs of the main mono- and polyfunctional harmonies.

At the same time, the ratio of texture elements is characterized by the "multivaluedness of connections: a single sound can belong to a clearly expressed layer, but at the same time it can be a part of another (along with the main one) textural system — a "network" or "veil" of quite convex sounds, as if dispersed throughout the material" [3]. The theatricalization and psychologization of texture are combined with likening of the development of individual lines and internal processes with unexpected "thought invasions", interruptions, their parallel existence.

One or another kind of polyphonic technique, on the one hand, is open for different interpretations and sometimes allows a polar opposite interpretation through the accentuation of certain technical properties. On the other hand, there is a psychologization of polyphonic techniques, the saturation of specific linguistic properties (temporal distance and interval step, direction, number of conducts, etc.) with bright semantic meanings, introduction into the context of the reflection of the hero's fate.

But the most important thing is that "due to the huge potential thematic possibilities, in the process of the historical evolution of the musical language the texture began to perform thematic functions more and more. This is due to the general tendency to increase the texture colorfulness, multidimensionality, strengthening of genre and visual moments [4].

In addition, the texture is also complicated due to the change of homophonic harmony into homophonicpolyphonic one, or homophonic with elements of sub-voice. Such textural process that does not have non-melodic voices, each one contains a potential melodic opening in its folded form and can at some point perform the most important thematic function it gives the texture a special fineness and delicate complexity.

The romantic art of "textural architecture" (M. Skrebkova-Filatova) generates two tendencies directed differently. The first one is directed to the polarization of voices, the isolation of their textural function, the identification of the leading melos. The second tendency is directed to synthetic combination of textured types and combination of different textural units. Each of these processes is carried out not only as a vector of the style's general orientation, but can be also implemented within one work.

Preservation of a single textural model throughout the statement is quite a rare phenomenon and is associated with a special imaginative task (creating a feeling of absorption by one feeling, spellbound contemplation, etc.). In most cases, after a few beats, a "textural deviation" or "textural modulation" occur: the type of narration and harmony change, their rethinking takes place.

Quite often in the Late Romantic texture a combination of two or more melodic lines occurs. Let us refer to one of the examples that reveals the patterns of Late Romantic texture. The final of Mahler's Symphony No. 3 is a solemn exalted hymn that reflects "the exalted contemplation of harmony that turns into a strong inner excitement in the face of all" [5], see "Fig. 2" and "Fig. 3", warm timbre of violins emphasizes the noble cantilena, the smoothness of its lines, the spirituality and depth of the statement. The final of the grandiose symphonic concept is not accidentally created as a polymelodic texture in which the melodic beginning is "shared" with all voices:
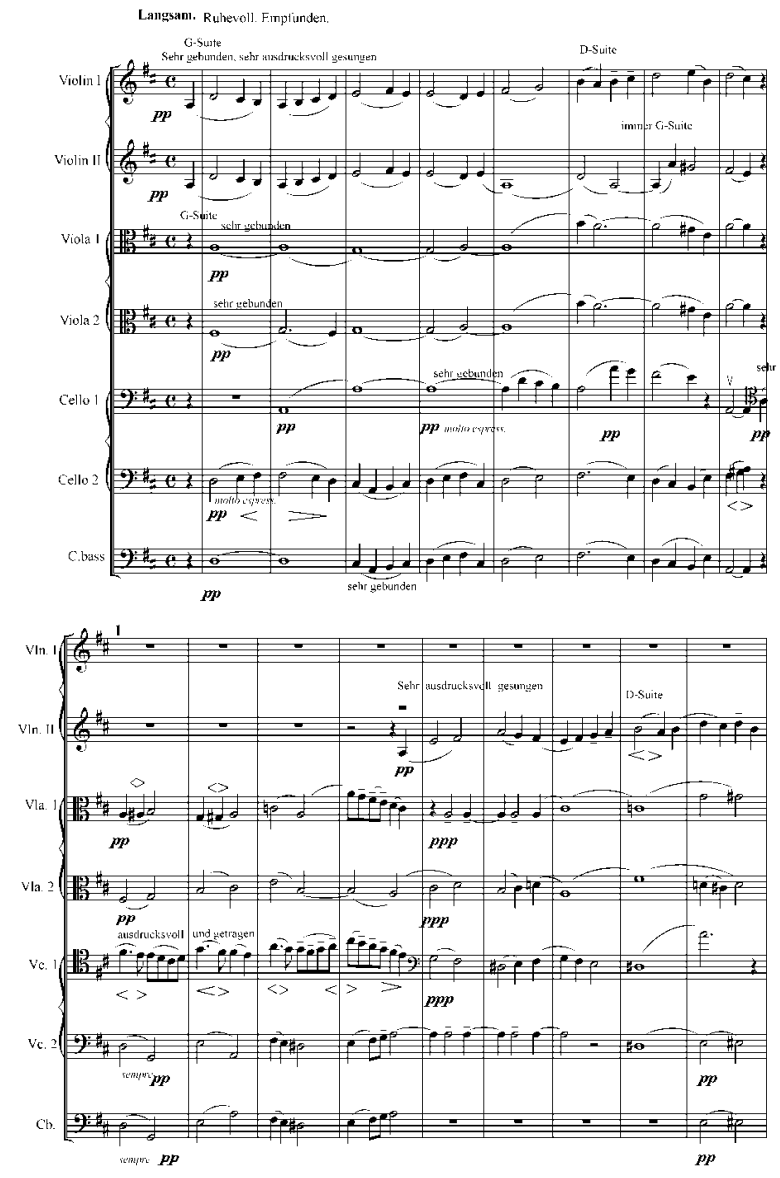

Fig. 2. G. Mahler. Symphony No. 3. P. IV. 

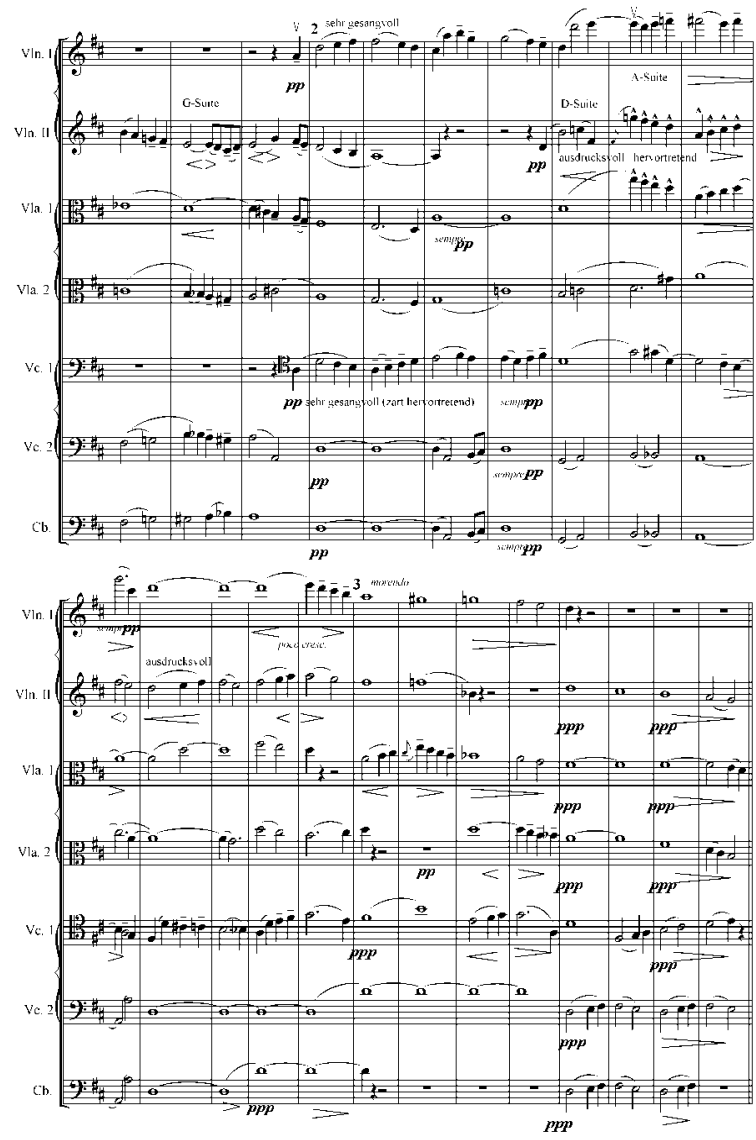

Fig. 3. G. Mahler. Symphony No. 3. P. IV.

The melody traditionally placed in the upper voice is initially supported by a bass patterned on the contour doublevoiced example, the inner voices from the seventh beat become more independent, and they borrow the function of the leading melodic voice from the ninth beat. Further movement occurs along the path of increasing the number of melodic lines and sub-voices, as well as enhancing the intonational characteristics of each voice. The initial almost absolute conjunction is quite quickly replaced by tritone, quint, nonet with bright bounces. The accuracy in the ratio of lines is striking when, in accordance with the complementarity principle, each intonational event (bounce, syncope, chromaticism) is balanced by the smoothest and steady movement in the other part, and each of them at a certain moment assumes the function of the most thematically concentrated melody.

The specificity of Late Romantic texture that is so obvious in this example, lies in frequent change of the type of narration, in the change in the number of voices and their functions, in the constant oscillation in the topography and background, making the musical material amazingly lively, breathing and surprisingly accurate in conveying the subtlest emotional colors.

It is also important to note that the most important principle of polyphony is embodied in the correlation of melodic lines - different significance gradations of the thematic material, the change in functions depending on the texture location. The ratio of melodic material can often be likened to the correlation of themes in multi-theme fugues and the correlation between the theme and counterthemes. Thus, there is a mixed harmony combining the polyphonic ratio of the leading voices and the homophonic division into melodic relief and background accompaniment.

The phenomenon that is at first glance very similar to the contrasting polyphony of operas and romantic sonatasymphonic cycles. However, in most cases the similarity is external. First, contrasting polyphony is often the result of combining the images that were previously exposed sequentially. Secondly, in many cases, it's not different characters that are emphasized, but different angles of a single statement. The emerging dialogue becomes a reflection of the intonational plot formation, the multiplicity of the view of the world.

Melodies don't have a single figurative "theatrical characters", the number of voices, correlation between them and their function is changing constantly. Thus, it is often not about the multi-theme (or contrast) polyphony, but about a single theme, a whole statement built on two or many voices.

A special feature of the Late Romantic style is the texture variation. The technique itself was brilliantly worked out in past eras in the genre of variations. The cycles of French harpsichordists and masters of Viennese Classicism demonstrated the truly inexhaustible ingenuity of theme transformations. However, the forms and methods found were not only transferred to a different compositional and genre sphere (first of all, to the recapitulation sections of the three-part and sonata forms and verse-variational sections of miniatures), but are also supplemented with a number of specific techniques. The technique of textural variation indicates with the greatest evidence that texture is not something external, complementary for the leading voice, but it is created as revealing of its immanent properties. That is why any change in the musical material causes a metamorphosis.

\section{CONCLUSION}

As a result, similar to how hard boundaries are overcome in harmony and syntax (tendency to "endless melody", periodicity overcoming, avoidance of cadences, etc.), a similar phenomenon is observed in the interpretation of texture boundaries: melody and bass, severely restricting the limits of the material, lose the course set on location, and sometimes one of the components may be absent. Thus, the "framework of integrity" (B. Uspenskiy) that gave the work a perfect appearance, disappears, giving way to a "stream", "magma" dissolving and absorbing the melodic lines.

There is a combination of functions in mixed harmonies - the leading melody can be simultaneously a subject, harmonic voices can be answers, the supporting voice is transformed into a counterpoint developed melodic line, and various "textural modulations" occur. Probably, this phenomenon is one of many mysteries of Late Romantic art - elusive transitions and mixing contribute to the 
multivalued perception of all means of the musical language, including texture.

Such a complex and multivalue phenomenon which the Late Romantic texture can be rightly considered cannot be forced into the "Procrustean bed" of classifications, schemes and rules. The unpredictability and variability, subtlety and delicacy of mixing and transitions in the textural appearance of the statements of this epoch lead to countless associations, spatial, descriptive, psychological analogies which turns the study of musical material, its inner life into an entertaining dialogical process where we can find discoveries, but not absolute truth.

\section{REFERENCES}

[1] M.S. Skrebkova-Filatova. Texture in Music. Art Opportunities. Structure. Functions / M.S. Skrebkova-Filatova; Moscow State Conservatory, Department of Music Theory. Moscow,Muzyka, $1985, \mathrm{p}, 222$.

[2] I.A.Barsova. Gustav Mahler's Symphonies / I. Barsova. Moscow,SovetskiyCompozitor, 1975, p. 337.

[3] Skrebkova-Filatova. op. cit., p. 91.

[4] E.V.Nazaykinskiy. The Logics of a Musical Composition / E.V. Nazaykinskiy. Moscow,Muzyka, 1982,p. 180.

[5] Barsova. op. cit., p. 128. 